\title{
Tracking brain injury and disability
}

B rain injury is often described as a "silent epidemic," but is that because we' re not listening?

The Brain Injury Association of Canada estimates that thousands of Canadians incur traumatic brain injuries each year, the majority being young adults. But many will not receive adequate treatment or followup for these injuries, particularly those with repeat mild concussions, because there's no consistent way to track the incidence and impact of such trauma over time.

"People aren't reporting it, and if they do, those reports gets buried in their medical records, so unless they remember every time they've had a concussion, there's no way to track backwards when behavioural and other changes happen," says association Executive Director Harry Zarins.

He's calling for the creation of a national registry that would track both cases of injury and possible related disability. For example, "it would take into consideration a person's concussion at age 7 from hockey, and again at age 10 playing football, as well as any subsequent misbehaviour or drop in academic performance."

According to the American Academy of Orthopaedic Surgeons, mild traumatic brain injury is linked to increased risk for persistent cognitive impairment, depression, anxiety, posttraumatic stress disorder, and other financial, social and emotional challenges.

Studies conducted in Ontario reveal that $23 \%$ of people in forensic psychiatric settings, $44 \%$ of provincial prison inmates, and nearly half of Toronto's homeless population report a history of brain injury. Surveys done internationally show higher proportions still, says Zarins, but it's impossible to know what to make of these links without lifetime surveillance.

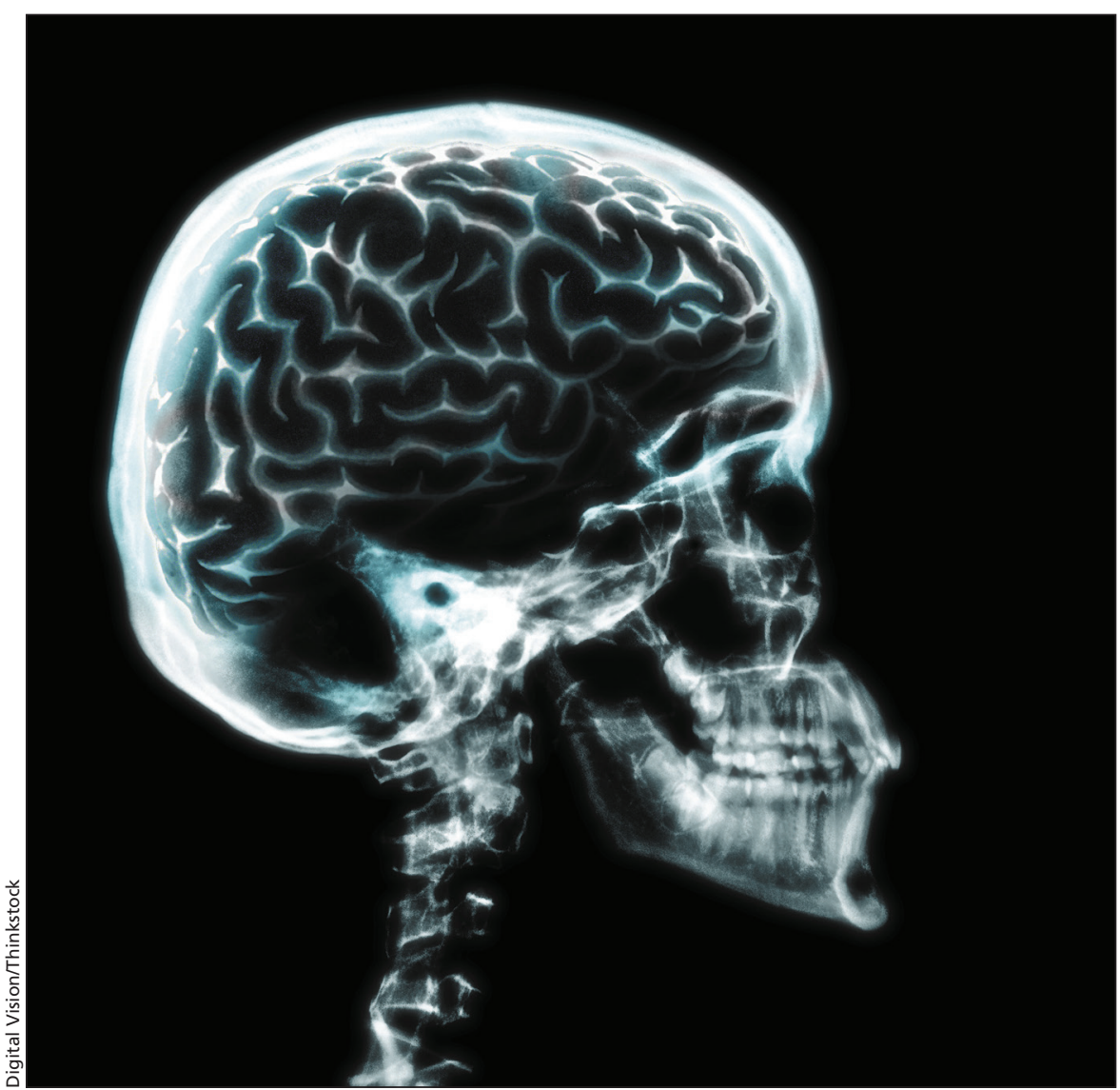

Many cognitive and behavioural problems may stem from a bump to the head, but we won't know for sure without national surveillance of brain injuries, say experts.

There's already some discussion of creating an Ontario registry, says Dr. Lisa Fischer, chair of the Sport and Exercise Medicine Program Committee at the College of Family Physicians of Canada. Increasing media attention to concussion in sports has also raised public awareness of brain injury more generally, she says. But the cost, time and infrastructure involved in establishing and maintaining a registry are major hurdles.

"It's hard to ask physicians in busy emergency departments to submit data to yet another registry - they need additional resources and personnel to do that," says Fischer.
Meanwhile, it would be challenging to mine data from existing electronic medical records because "there isn't one system that communicates uniformly across the province, let alone the country," she says. "The big picture just isn't there."

Long waits for specialist assessment and a dearth of objective diagnostic tools for concussion also complicate the picture, says Zarins. "That's the scary part of it, because as a society we're not prepared to deal with the spectrum of disabilities associated with brain injury." — Lauren Vogel, CMAJ

CMAJ 2014. DOI:10.1503/cmaj.109-4854 